https://doi.org/10.15407/ujpe64.12.1148

V.M. SIMULIK, ${ }^{1}$ I.O. GORDIEVICH ${ }^{2}$

${ }^{1}$ Institute of Electron Physics,

Nat. Acad. of Sci. of Ukraine

(21, Universytets'ka Str., Uzhgorod 88017, Ukraine; e-mail: vsimulik@gmail.com)

2 MIRTEK

(Stavropol, Russian Federation)

\title{
SYMMETRIES OF RELATIVISTIC HYDROGEN ATOM ${ }^{1}$
}

\begin{abstract}
The Dirac equation in the external Coulomb field is proved to possess the symmetry determined by 31 operators, which form the 31-dimensional algebra. Two different fermionic realizations of the SO(1,3) algebra of the Lorentz group are found. Two different bosonic realizations of this algebra are found as well. All generators of the above-mentioned algebras commute with the operator of the Dirac equation in an external Coulomb field and, therefore, determine the algebras of invariance of such Dirac equation. Hence, the spin $s=(1,0)$ Bose symmetry of the Dirac equation for the free spinor field, proved recently in our papers, is extended here for the Dirac equation interacting with an external Coulomb field. A relativistic hydrogen atom is modeled by such Dirac equation. We are able to prove for the relativistic hydrogen atom both the fermionic and bosonic symmetries known from our papers in the case of a noninteracting spinor field. New symmetry operators are found on the basis of new gamma matrix representations of the Clifford and $S O(8)$ algebras, which are known from our recent papers as well. Hidden symmetries were found both in the canonical Foldy-Wouthuysen and covariant Dirac representations. The found symmetry operators, which are pure matrix ones in the Foldy-Wouthuysen representation, become non-local in the Dirac model.
\end{abstract}

Keywords: Dirac equation, Coulomb interaction, hydrogen atom, relativistic quantum mechanics, symmetry.

\section{Introduction}

Symmetry studies of the equations for a hydrogen atom originate from the non-relativistic case. The SO(4) symmetry of the non-relativistic Schrödinger equation for a hydrogen atom was found by V. Fock [1], see also [2].

A relativistic hydrogen atom is modeled here by the Dirac equation in an external Coulomb field

$$
\left(i \partial_{0}-\widehat{H}\right) \psi(x)=0 ; \quad \widehat{H} \equiv \gamma^{0} \boldsymbol{\gamma} \cdot \mathbf{p}+\gamma^{0} m-\frac{Z e^{2}}{|\mathbf{x}|},
$$

where $x \in \mathrm{M}(1,3), \partial_{\mu} \equiv \partial / \partial x^{\mu}, Z=1, \mu=\overline{0,3}, j=$ $=1,2,3$, and $\mathrm{M}(1,3)=\left\{x \equiv\left(x^{\mu}\right)=\left(x^{0}=t, \mathbf{x} \equiv\right.\right.$ $\left.\left.\equiv\left(x^{j}\right)\right)\right\}$ is the Minkowski space-time, the 4-component function $\psi(x)$ belongs to the rigged Hilbert space $\mathrm{S}^{3,4} \subset \mathrm{H}^{3,4} \subset \mathrm{S}^{3,4 *}$.

Note that, due to a special role of the time variable $x^{0}=t \in\left(x^{\mu}\right)$ (in obvious analogy with non-relati-

(C) V.M. SIMULIK, I.O. GORDIEVICH, 2019

\section{8}

vistic theory), one can use the quantum-mechanical rigged Hilbert space (2) in the general consideration. Here, the Schwartz test function space $S^{3,4}$ is dense in the Schwartz generalized function space $S^{3,4 *}$, and $\mathrm{H}^{3,4}$ is the quantum-mechanical Hilbert space of 4component functions over $\mathrm{R}^{3} \subset \mathrm{M}(1,3)$.

In order to finish with notations, assumptions, and definitions, we note that the system of units $\hbar=c=1$ is chosen, the metric tensor in the Minkowski spacetime $\mathrm{M}(1,3)$ is given by

$g^{\mu \nu}=g_{\mu \nu}=g_{\nu}^{\mu},\left(g_{\nu}^{\mu}\right)=\operatorname{diag}(1,-1,-1,-1) ;$

$x_{\mu}=g_{\mu \nu} x^{\mu}$, and the summation over the twice repeated indices is implied. The Dirac $\gamma$ matrices are taken in the standard Dirac-Pauli representation

$\gamma^{0}=\left|\begin{array}{ll}\mathrm{I} & 0 \\ 0 & -\mathrm{I}\end{array}\right|, \quad \gamma^{\ell}=\left|\begin{array}{ll}0 & \sigma^{\ell} \\ -\sigma^{\ell} & 0\end{array}\right|, \quad \ell=1,2,3$,

1 This work is based on the results presented at the XI BolyaiGauss-Lobachevskii (BGL-2019) Conference: Non-Euclidean, Noncommutative Geometry and Quantum Physics.

ISSN 2071-0194. Ukr. J. Phys. 2019. Vol. 64, No. 12 
where the Pauli matrices are given by

$\sigma^{1}=\left|\begin{array}{ll}0 & 1 \\ 1 & 0\end{array}\right|, \sigma^{2}=\left|\begin{array}{ll}0 & -i \\ i & 0\end{array}\right|, \sigma^{3}=\left|\begin{array}{ll}1 & 0 \\ 0 & -1\end{array}\right|$.

Below, we will prove that the Dirac equation in the external Coulomb field (1) possesses the symmetry determined by 31 operators, which form the 31 -dimensional algebra $\mathrm{SO}(6) \oplus i \gamma^{0} \times \mathrm{SO}(6) \oplus i \gamma^{0}$. Two different fermionic $\mathrm{D}(1 / 2,0) \oplus(0,1 / 2)$ representations of the $\mathrm{SO}(1,3)$ algebra of the Lorentz group are found. Two different bosonic tensor-scalar $\mathrm{D}(1,0) \oplus(0,0)$ and vector $\mathrm{D}(1 / 2,1 / 2)$ representations of this algebra are found as well. The corresponding generators of the above-mentioned algebras commute with the operator of the Dirac equation in the external Coulomb field (1) and, therefore, determine the hidden symmetries (algebras of invariance) of the Dirac equation.

At first, we consider the known symmetries of the Dirac equation (1). Then we present the mathematical tools, which are necessary for our investigations, and, finally, the list of different hidden symmetries of a relativistic hydrogen atom.

\section{Known Symmetries of the Dirac Equation in an External Coulomb Field}

The first four constants of motion (symmetry operators, which commute with the operator of the Dirac equation) for Eq. (1) were found in [3], where Eq. (1) was derived. They are three components of the vector $\mathbf{J}=\left(J^{1}, J^{2}, J^{3}\right)$ of the total angular momentum

$\mathbf{J}=\mathbf{L}+\mathbf{s}, \quad \mathbf{L} \equiv \mathbf{x} \times \mathbf{p}, \quad \mathbf{s} \equiv \frac{\mathbf{1}}{\mathbf{2}}\left|\begin{array}{cc}\boldsymbol{\sigma} & 0 \\ 0 & \boldsymbol{\sigma}\end{array}\right|$,

where $\mathbf{L}$ is the orbital angular momentum, $\mathbf{s}$ is the spin- $1 / 2$ angular momentum, and $K$ is the additional constant of motion:

$K=\gamma^{0}(2 \mathbf{s} \cdot \mathbf{L}+1), \quad K^{2}=\mathbf{J}^{2}+\frac{1}{4}$.

Next symmetry operator is the Johnson-Lippman constant of motion [4]

$D=2 \mathbf{s} \cdot \frac{\mathbf{x}}{|\mathbf{x}|}+\frac{1}{m Z e^{2}} K \gamma^{4}\left(\widehat{H}-\gamma^{0} m\right)$,

$D^{2}=1+\left(\frac{\widehat{H}^{2}}{m^{2}}-1\right) \frac{K^{2}}{Z^{2} e^{4}}$. It commutes with the operator $\left(i \partial_{0}-\widehat{H}\right)$ of the Dirac equation and anticommutes ISSN 2071-0194. Ukr. J. Phys. 2019. Vol. 64, No. 12 with the Dirac symmetry operator (7). Here, the antiHermitian $\gamma^{4}=\gamma^{0} \gamma^{1} \gamma^{2} \gamma^{3}$ instead of the Hermitian $\gamma^{5}$ of other authors is used. Note that work [4] on the excellent result (8) was published as a brief remark 1/10 of a page in size containing only the single formula for $D$ from (8).

After that, the way to the $\mathrm{SO}(4)$ symmetry of a relativistic hydrogen atom was direct. This symmetry was found in [5] and [6] (as for the consideration in [7], see the comments in [8]). Thus, the $\mathrm{SO}(4)$ symmetry of the Dirac equation (1) for a hydrogen atom is given by the six operators

$\mathbf{I}=\mathbf{J}+\mathbf{T}, \quad \mathbf{R}=\mathbf{J}-\mathbf{T}$,

where $\mathbf{J}$ is known from (6), and components of $\mathbf{T}=$ $=\left(T^{1}, T^{2}, T^{3}\right)$ have the form

$T^{1}=\frac{D}{2 \sqrt{D^{2}}}, \quad T^{2}=\frac{i D K}{2 \sqrt{D^{2} K^{2}}}, \quad T^{3}=\frac{K}{2 \sqrt{K^{2}}}$.

In work [5], the object $\mathbf{T}=\left(T^{1}, T^{2}, T^{3}\right)$ (10) was called the Lenz spin- $1 / 2$ vector operator. The notations used in (10) are explained in (7) and (8) above.

The Pauli-Gürsey operators [9] and [10]

$s^{01}=\frac{i}{2} \gamma^{2} \hat{C}, \quad s^{02}=\frac{1}{2} \gamma^{2} \hat{C}, \quad s^{12}=-\frac{i}{2}$,

where $\hat{C}$ is the operator of complex conjugation, $\hat{C} \psi=\psi^{*}$ (the operator of involution in the space $\left.\mathrm{H}^{3,4}\right)$, determine according to [11], the $\mathrm{SO}(1,2) \subset$ $\subset \mathrm{SO}(1,3)$ algebra of invariance of the Dirac equation in the form $\left(i \gamma^{\mu} \partial_{\mu}-m+\frac{e^{2}}{|\mathbf{x}|}\right) \psi(x)=0$.

In [12], the symmetry of a relativistic hydrogen atom in the form of $g l(8, \mathrm{R})$ algebra has been found. The stationary case of the Dirac equation (1) was considered, and the discrete transformation were used.

The author of [13] considered another problem. The quasipotential two-particle model was presented for the description of a spinless relativistic hydrogen atom. The $\mathrm{O}(4)$ symmetry and its breaking were investigated.

\section{Briefly on the Gamma Matrix Representations of the Real Clifford and $\mathrm{SO}(8)$ Algebras}

In our long-term investigations of the mapping of the Maxwell theory on the Dirac theory, we also studied 
the different exotic representations of the CliffordDirac algebra, which were useful in the description of the Maxwell theory in the notations taking from the formalism of the Dirac spinor field (see, e.g., [14] and [15]). Step-by-step, we came to the idea of a generalization of the Clifford-Dirac algebra in order to describe the bosonic features of the Dirac theory and the fermionic features of the Maxwell theory as well.

Recently (see, e.g., [16]), we put the gamma matrix representations of the Clifford algebra $C \ell^{\mathbb{R}}(0,6)$ and the Lie algebra $\mathrm{SO}(8)$ into consideration, which are defined over the field of real numbers. The comparison with the well-known gamma matrix representations of the algebras $C \ell^{\mathbb{C}}(1,3)$ (in the physical literature, the Clifford-Dirac algebra) and $\mathrm{SO}(1,5)$ defined over the field of complex numbers demonstrates that the new representations contain much more useful elements and perspectives for applications. The appealing to the gamma matrix representations of the Clifford algebra $C \ell^{\mathbb{R}}(0,6)$ and the Lie algebra $\mathrm{SO}(8)$ made it possible (see, e.g., [17] and [18]) to find the hidden symmetries of the free (non-interacting) Dirac equation. The Bose symmetries were found as well [19]. Below, we will use these new algebraic objects [16] for the problem of finding the hidden symmetries of a relativistic hydrogen atom. The necessary part of the consideration from [16] is presented in a compact version.

Consider the fact that seven $\gamma$ matrices $\gamma^{1}, \gamma^{2}, \gamma^{3}$, $\gamma^{4}=\gamma^{0} \gamma^{1} \gamma^{2} \gamma^{3}, \gamma^{5}=\gamma^{1} \gamma^{3} \hat{C}, \gamma^{6}=i \gamma^{1} \gamma^{3} \hat{C}, \gamma^{7}=i \gamma^{0}$

where $\gamma^{\mu}$ matrices are given in (4), and the operator $\hat{C}$ is defined in text after formulas (11), satisfy the anticommutation relations

$\gamma^{\mathrm{A}} \gamma^{\mathrm{B}}+\gamma^{\mathrm{B}} \gamma^{\mathrm{A}}=-2 \delta^{\mathrm{AB}}, \quad \mathrm{A}, \mathrm{B}=\overline{1,7}$,

of the Clifford algebra generators over the field of real numbers. Due to the evident fact that only six operators of (12) are linearly independent, $\gamma^{4}=$ $=-i \gamma^{7} \gamma^{1} \gamma^{2} \gamma^{3}$, it is the representation of the Clifford algebra $C \ell^{\mathbb{R}}(0,6)$ of the dimension equal to $2^{6}=64$.

The first 16 operators are given in Table 1 of [16], the next 16 ones can be found from them with the help of the multiplication by imaginary unit $i=\sqrt{-1}$. Last 32 operators follow from first 32 ones with the help of the multiplication by the operator $\hat{C}$ of com- plex conjugation. Thus, if we introduce the notation "stCD" ("st" and "CD" are taken from "standard Clifford-Dirac") for the set of 16 matrices from Table 1 in [16], then the set of 64 elements of the $C \ell^{\mathbb{R}}(0,6)$ algebra representation is given by

$$
\{(\operatorname{stCD}) \cup i \times(\operatorname{stCD}) \cup \hat{C} \times(\operatorname{stCD}) \cup i \hat{C} \times(\text { stCD })\} .
$$

The equalities $\quad \gamma^{4} \equiv \prod_{\mu=0}^{3} \gamma^{\mu} \rightarrow \prod_{\bar{\mu}=0}^{4} \gamma^{\bar{\mu}}=-\mathrm{I}$ known from the standard Clifford-Dirac algebra $C \ell^{\mathbb{C}}(1,3)$ and the anticommutation relations (13) of the $C \ell^{\mathbb{R}}(0,6)$ algebra for the matrices $\gamma^{\mathrm{A}}(12)$ lead to the validity of the following extended equalities:

$\gamma^{7} \equiv-\prod_{\underline{\mathrm{A}}=1}^{6} \gamma^{\underline{\mathrm{A}}} \rightarrow \prod_{\mathrm{A}=1}^{7} \gamma^{\mathrm{A}}=\mathrm{I}, \quad \gamma^{5} \gamma^{6}=i$.

Operators (12) generate also 28 matrices

$s^{\widetilde{\mathrm{A}} \widetilde{\mathrm{B}}}=\left\{s^{\mathrm{AB}}=\frac{1}{4}\left[\gamma^{\mathrm{A}}, \gamma^{\mathrm{B}}\right], s^{\mathrm{A} 8}=-s^{8 \mathrm{~A}}=\frac{1}{2} \gamma^{\mathrm{A}}\right\}$,

$\widetilde{\mathrm{A}}, \widetilde{\mathrm{B}}=\overline{1,8}$, which satisfy the commutation relations of the Lie algebra $\mathrm{SO}(8)$

$\left[s^{\widetilde{\mathrm{A}} \widetilde{\mathrm{B}}}, s^{\widetilde{\mathrm{C}} \widetilde{\mathrm{D}}}\right]=\delta^{\widetilde{\mathrm{A}} \widetilde{\mathrm{C}}} s^{\widetilde{\mathrm{B}} \widetilde{\mathrm{D}}}+\delta^{\widetilde{\mathrm{C}} \widetilde{\mathrm{B}}} s^{\widetilde{\mathrm{D}} \widetilde{\mathrm{A}}}+\delta^{\widetilde{\mathrm{B}} \widetilde{\mathrm{D}}} s^{\widetilde{\mathrm{A}} \widetilde{\mathrm{C}}}+\delta^{\widetilde{\mathrm{D}} \widetilde{\mathrm{A}}} s^{\widetilde{\mathrm{C}} \widetilde{\mathrm{B}}}$

It is evident that we have the algebra over the field of real numbers as well. Furthermore, it is evident that 28 elements (15) of $\mathrm{SO}(8)$ do not form any Clifford algebra and do not form any subalgebra of the Clifford algebra. It is a mathematical object independent of the Clifford algebra. Note that the anti-Hermitian realization of the $\mathrm{SO}(8)$ operators is chosen here (as in (12) for the gamma matrices) (for the reasons, see, e.g., [20] and [21] or [22]).

The explicit form of 28 elements of the $\gamma$ matrix representation of the $\mathrm{SO}(8)$ algebra is given in Table 3 of [16]. The wonderful feature of this gamma matrix representation of the algebra $\mathrm{SO}(8)$ is as follows. Here, (i) two subsets $\left(s^{23}, s^{31}, s^{12}\right)$ and $\left(s^{45}, s^{64}, s^{56}\right)$ of the operators $s^{\widetilde{\mathrm{A}}}$ from (15) determine two different sets of $\mathrm{SU}(2)$ spin $1 / 2$ generators, (ii) commute with each other and (iii) commute with the operator of the Dirac equation in the Foldy-Wouthuysen representation [23].

ISSN 2071-0194. Ukr. J. Phys. 2019. Vol. 64, No. 12 


\section{New Symmetries}

\section{of a Relativistic Hydrogen Atom}

Below, we present both the symmetries of the FoldyWouthuysen equation [23] in the external Coulomb field

$$
\left(i \partial_{0}-\gamma^{0} \omega+\frac{e^{2}}{|\mathbf{x}|}\right) \phi(x)=0 ; \quad \omega \equiv \sqrt{-\Delta+m^{2}},
$$

$x \in \mathrm{M}(1,3), \phi \in\left\{\mathrm{S}^{3,4} \subset \mathrm{H}^{3,4} \subset \mathrm{S}^{3,4 *}\right\}$, and, of course, the symmetries of the Dirac equation (1) in such external field as well.

The first application of the matrix representations of the algebras $C \ell^{\mathbb{R}}(0,6)$ and $\mathrm{SO}(8)$ is the symmetry analysis (the search for groups and algebras, with respect to which the equation is invariant). It is easy to understand that the Foldy-Wouthuysen representation [23] is preferable for such analysis. Indeed, in this representation, one must calculate the commutation relations of possible pure matrix symmetry operators from (15) only with two elements of the operator of the Foldy-Wouthuysen equation (17): $\gamma^{0}$ and $i$. After the determination of the symmetries of the Foldy-Wouthuysen equation, one can find the symmetries of the Dirac equation on the basis of the inverse Foldy-Wouthuysen transformation [23]. Note that, after such transformation, only a small part of the symmetry operators will be purely matrix ones, the main part of operators will contain the nonlocal operator $\omega \equiv \sqrt{-\Delta+m^{2}}$ and the functions of it.

Now, we can start the consideration of the new symmetries of a relativistic hydrogen atom. The beginning was suggested in [24]. The fundamental assertions are as follows.

(i) The gamma matrix representation of the subalgebra $\mathrm{SO}(6)$ of the algebra $\mathrm{SO}(8)$, which is formed by the operators

$$
\left\{s^{\breve{\mathrm{A}} \breve{\mathrm{B}}}\right\}=\left\{s^{\breve{\mathrm{A}} \breve{\mathrm{B}}} \equiv \frac{1}{4}\left[\gamma^{\breve{\mathrm{A}}}, \gamma^{\breve{\mathrm{B}}}\right]\right\}, \quad \breve{\mathrm{A}}, \breve{\mathrm{B}}=\overline{1,6},
$$

determines the algebra of invariance of the Dirac equation in the Foldy-Wouthuysen representation $\left(\partial_{0}+i \gamma^{0} \omega-\frac{e^{2}}{|\mathbf{x}|}\right) \phi(x)=0$ (in (18) six matrices $\left\{\gamma^{\breve{A}}\right\}=\left\{\gamma^{1}, \gamma^{2}, \gamma^{3}, \gamma^{4}, \gamma^{5}, \gamma^{6}\right\}$ are known from (12)).

(ii) On the basis of $\mathrm{SO}(6)$ (18), the 31-dimensional gamma matrix representation of the Lie algebra $\mathrm{SO}(6) \oplus i \gamma^{0} \mathrm{SO}(6) \oplus i \gamma^{0}$ is constructed, which is formed by the elements from $C \ell^{\mathbb{R}}(0,6)$ and is the maximal pure matrix algebra of invariance of the Dirac equation in the Foldy-Wouthuysen representation $\left(\partial_{0}+\right.$ $\left.+i \gamma^{0} \omega-\frac{e^{2}}{|\mathbf{x}|}\right) \phi(x)=0$.

(iii) The Dirac equation in an external Coulomb field (1) is invariant with respect to the 31-dimensional gamma matrix representation of the algebra $\widetilde{\mathrm{SO}}(6) \oplus i \widetilde{\gamma}^{0} \widetilde{\mathrm{SO}}(6) \oplus i \widetilde{\gamma}^{0}$, where the representation of the algebra $\widetilde{\mathrm{SO}}(6)$ is given in the form of (18) with gamma operators found from matrices (12) by the inverse Foldy-Wouthuysen transformation [23]. The resulting operators are given by

$\widetilde{\gamma}=\gamma \frac{-\gamma \cdot \nabla+m}{\omega}+\mathbf{p} \frac{-\gamma \cdot \nabla+\omega+m}{\omega(\omega+m)}$,

$\widetilde{\gamma}^{4}=\gamma^{4} \frac{-\gamma \cdot \nabla+m}{\omega}, \quad \widetilde{\gamma}^{5}=\widetilde{\gamma}^{1} \widetilde{\gamma}^{3} \widetilde{C}$,

$\widetilde{\gamma}^{6}=i \widetilde{\gamma}^{1} \widetilde{\gamma}^{3} \widetilde{C}, \quad \widetilde{\gamma}^{7}=i \widetilde{\gamma}^{0}, \quad \widetilde{\gamma}^{0}=\gamma^{0} \frac{-\gamma \cdot \nabla+m}{\omega}$,

where $\widetilde{C}=\left(\mathrm{I}+2 \frac{i \gamma^{1} \partial_{1}+i \gamma^{2} \partial_{2}}{\sqrt{2 \omega(\omega+m)}}\right) \hat{C}$, and $\omega \equiv \sqrt{-\triangle+m^{2}}$. These formulas give the images of the gamma matrices (12) in the Dirac representation after fulfilling the inverse Foldy-Wouthuysen transformation.

Thus, the $\widetilde{\mathrm{SO}}(6) \oplus i \widetilde{\gamma}^{0} \widetilde{\mathrm{SO}}(6) \oplus i \widetilde{\gamma}^{0}$ algebra is found from $\mathrm{SO}(6) \oplus i \gamma^{0} \mathrm{SO}(6) \oplus i \gamma^{0}$ on the basis of the inverse Foldy-Wouthuysen transformation [23]. For the Dirac equation, only a part of this algebra is a purely matrix one, other elements contain the operator $\omega \equiv$ $\equiv \sqrt{-\Delta+m^{2}}$.

Consider the symmetries of a relativistic hydrogen atom with respect to the Lorentz group. On the basis of $C \ell^{\mathbb{R}}(0,6)$ and $\mathrm{SO}(8)$, we can determine two different realizations of the $\mathrm{D}\left(0, \frac{1}{2}\right) \oplus\left(\frac{1}{2}, 0\right)$ representation of the Lie algebra of universal covering $\mathcal{L}=$ $=\mathrm{SL}(2, \mathrm{C})$ of the proper ortochronous Lorentz group $\mathrm{L}_{+}^{\uparrow}=\mathrm{SO}(1,3)=\left\{\Lambda=\left(\Lambda_{\nu}^{\mu}\right)\right\}$, with respect to which the equation $\left(\partial_{0}+i \gamma^{0} \omega-\frac{e^{2}}{|\mathbf{x}|}\right) \phi(x)=0$ is invariant:

$s_{\mathrm{I}}^{\mu \nu}=\left\{s_{\mathrm{I}}^{0 k}=\frac{i}{2} \gamma^{k} \gamma^{4}, \quad s_{\mathrm{I}}^{k m}=\frac{1}{4}\left[\gamma^{k}, \gamma^{m}\right]\right\}$,

$\gamma^{4} \equiv \gamma^{0} \gamma^{1} \gamma^{2} \gamma^{3}, \quad(k, m=\overline{1,3})$,

$s_{\mathrm{II}}^{01}=-\frac{i}{2} \gamma^{2} \hat{C}, s_{\mathrm{II}}^{02}=-\frac{1}{2} \gamma^{2} \hat{C}, s_{\mathrm{II}}^{03}=\frac{1}{2} \gamma^{0}$,

$s_{\mathrm{II}}^{23}=-\frac{1}{2} \gamma^{0} \gamma^{2} \hat{C}, s_{\mathrm{II}}^{31}=\frac{i}{2} \gamma^{0} \gamma^{2} \hat{C}, s_{\mathrm{II}}^{12}=-\frac{i}{2}$.

Taking the combinations of operators (20) and (21), we construct the generators of bosonic representa- 
tions:

$$
\begin{array}{ll}
s_{\mathrm{TS}}^{\mu \nu}=\left\{s_{\mathrm{TS}}^{0 k}=s_{\mathrm{I}}^{0 k}+s_{\mathrm{II}}^{0 k},\right. & \left.s_{\mathrm{TS}}^{m n}=s_{\mathrm{I}}^{m n}+s_{\mathrm{II}}^{m n}\right\}, \\
s_{\mathrm{V}}^{\mu \nu}=\left\{s_{\mathrm{V}}^{0 k}=-s_{\mathrm{I}}^{0 k}+s_{\mathrm{II}}^{0 k},\right. & \left.s_{\mathrm{V}}^{m n}=s_{\mathrm{TS}}^{m n}\right\},
\end{array}
$$

where $s_{\mathrm{TS}}^{\mu \nu}$ and $s_{\mathrm{V}}^{\mu \nu}$ are the generators of the tensor-scalar $\mathrm{D}(1,0) \oplus(0,0)$ and irreducible vector $\mathrm{D}\left(\frac{1}{2}, \frac{1}{2}\right)$ representations of the Lie algebra $\mathrm{SO}(1,3)$ of the Lorentz group $\mathcal{L}$, respectively, with respect to which the Foldy-Wouthuysen equation $\left(\partial_{0}+i \gamma^{0} \omega-\right.$ $\left.-\frac{e^{2}}{|\mathbf{x}|}\right) \phi(x)=0$ is invariant.

The anti-Hermitian operators of every of the sets (20), (21), or (22) satisfy the commutation relations of the Lie algebra $\mathrm{SO}(1,3)$ of the Lorentz group $\mathcal{L}$ :

$$
\left[s^{\mu \nu}, s^{\rho \sigma}\right]=-g^{\mu \rho} s^{\nu \sigma}-g^{\rho \nu} s^{\sigma \mu}-g^{\nu \sigma} s^{\mu \rho}-g^{\sigma \mu} s^{\rho \nu} .
$$

For the Dirac equation in the space of Dirac spinors $\{\psi\}$ (i.e., in the Pauli-Dirac representation), the form of the generators of the tensor-scalar $\mathrm{D}(1,0) \oplus(0,0)$ and irreducible vector $\mathrm{D}\left(\frac{1}{2}, \frac{1}{2}\right)$ representations of the Lie algebra $\mathrm{SO}(1,3)$ of the Lorentz group $\mathcal{L}$ is similar to (22) (with (20), (21)), but the gamma operators are too complicated in this case and are given by (19). The images of operators (20), (21), or (22) in the Dirac representation satisfy the commutation relations (23) as well.

In [15] and [18] for the free Dirac and Foldy-Wouthuysen equations, we used also the evident bosonic representation of (22), in which the Casimir operators are diagonal, and the proof of Bose properties is most convenient.

\section{Discussion and Summary}

A generalization of our results on the Bose symmetries of the free interactionless Dirac equation (see, e.g., [15] and the references therein) in the case of the presence of an external Coulomb field has been suggested. The hidden Fermi and Bose symmetries of a relativistic hydrogen atom are found.

Note that the physical picture of a hydrogen atom (the electron in the external Coulomb field) is related to the spin-1/2 Fermi symmetries. On the other hand, the physical picture of a hydrogen atom as the compound system of a proton and an electron is related to the total spin-1 (or zero) Bose symmetries.

The main result of this paper is putting two different bosonic tensor-scalar $\mathrm{D}(1,0) \oplus(0,0)$ and vector $\mathrm{D}(1 / 2,1 / 2)$ representations of the Lie algebra $\mathrm{SO}(1,3)$

\section{2}

of the Lorentz group $\mathcal{L}$ into consideration. With respect to them, the Dirac equation in an external Coulomb field is invariant. Two fermionic symmetries of this equation, given by different $\mathrm{D}(1 / 2,0) \oplus(0,1 / 2)$ representations of the $\mathrm{SO}(1,3)$ algebra of the Lorentz group, are found as well. The maximal pure matrix symmetry of the Foldy-Wouthuysen equation for a hydrogen atom is found, and the explicit forms of operators of the corresponding symmetry of the Dirac model are calculated.

1. V. Fock. Zur Theorie des Wasserstoffatoms. Z. Phys. 98, 145 (1935).

2. V. Bargmann. Zur Theorie des Wasserstoffatoms. Bemerkungen zur gleichnamigen Arbeit von V. Fock. Z. Phys. 99, 576 (1936).

3. P.A.M. Dirac. The quantum theory of the electron. Proc. Roy. Soc. Lond. A. 117, 610 (1928).

4. M.H. Johnson, B.A. Lippmann. Relativistic Kepler problem. Phys. Rev. 78, 329 (1950).

5. E. De Groot. The virial theorem and the Dirac H atom. Am. J. Phys. 50, 1141 (1982).

6. A.A. Stahlhofen. Algebraic solutions of relativistic Coulomb problems. Helv. Phys. Acta 70, 372 (1997).

7. J-L. Chen, D-L. Deng, M-G. Hu. SO(4) symmetry in the relativistic hydrogen atom. Phys. Rev. A. 77, 034102 (2008).

8. A.A. Stahlhofen. Comment on "SO(4) symmetry in the relativistic hydrogen atom". Phys. Rev. A. 78, 036101 (2008).

9. W. Pauli. On the conservation of the lepton charge. Nuovo Cim. 6, 204 (1957).

10. F. Gürsey. Relation of charge independence and baryon conservation to Pauli's transformation. Nuov. Cim. 7, 411 (1958).

11. I.Yu. Krivsky, V.M. Simulik. The Dirac equation and spin 1 representations, a connection with symmetries of the Maxwell equations. Theor. Math. Phys. 90, 265 (1992).

12. A.G. Nikitin. Superalgebras of symmetry operators for Coulomb and Aharonov-Bohm-Coulomb systems. In: Photon and Poincaré group (Nova Sci., 1999) [ISBN: 9781560727187].

13. Th.W. Ruijgrok. On the relativistic hydrogen atom. Acta Phys. Pol. 8743 (1976).

14. V.M. Simulik, I.Yu. Krivsky. Clifford algebra in classical electrodynamical hydrogen atom model. Adv. Appl. Cliff. Algebras 7, 25 (1997).

15. V.M. Simulik, I.Yu. Krivsky, I.L. Lamer. Bosonic symmetries, solutions and conservation laws for the Dirac equation with nonzero mass. Ukr. J. Phys. 58, 523 (2013).

16. V.M. Simulik. On the gamma matrix representations of $\mathrm{SO}(8)$ and Clifford algebras. Adv. Appl. Cliff. Algebras 28, 93 (2018).

ISSN 2071-0194. Ukr. J. Phys. 2019. Vol. 64, No. 12 
17. V.M. Simulik, I.Yu. Krivsky. On the extended real Clifford-Dirac algebra and new physically meaningful symmetries of the Dirac equation with nonzero mass. Dopov. NAN Ukr. No. 5, 82 (2010) (in Ukrainian).

18. I.Yu. Krivsky, V.M. Simulik. Fermi-Bose duality of the Dirac equation and extended real Clifford-Dirac algebra. Cond. Matt. Phys. 13, 43101 (2010).

19. V.M. Simulik, I.Yu. Krivsky, I.L. Lamer. Application of the generalized Clifford-Dirac algebra to the proof of the Dirac equation Fermi-Bose duality. TWMS J. App. Eng. Math. 3, 46 (2013).

20. V.M. Simulik, I.Yu. Krivsky. Bosonic symmetries of the Dirac equation. Phys. Lett. A. 375, 2479 (2011).

21. B. Wybourne. Classical Groups for Physicists (Wiley, 1974) [ISBN: 978-0471965053].

22. J. Elliott, P. Dawber. Symmetry in Physics (Macmillian Press, 1979), Vol. 1 [ISBN: 978-0333382707].

23. L.L. Foldy, S.A. Wouthuysen. On the Dirac theory of spin $1 / 2$ particles and its non-relativistic limit. Phys. Rev. 78, 29 (1950).

24. V.M. Simulik, I.O. Gordiyevich. On the symmetry of relativistic hydrogen atom and the Foldy-Wouthuysen representation. In: Abstracts of the Reports of the Intern. Conference of Young Scientists and Post-Graduates (Institute of Electron Physics, 2013).

Received 31.08.19
В.М. Симулик, I.О. Гордієвич

\section{СИМЕТРІЇ РЕЛЯТИВІСТСЬКОГО АТОМА ВОДНЮ}

$\mathrm{P}$ е з ю м е

Доведено, що рівняння Дірака у зовнішньому кулонівському полі має симетрію, що визначається 31 операторами, які утворюють 31-вимірну алгебру. Знайдено дві різні ферміонні реалізації алгебри $\mathrm{SO}(1,3)$ групи Лоренца. Отримано також дві бозонні реалізації цієї алгебри. Усі генератори цих алгебр комутують з оператором рівняння Дірака у зовнішньому кулонівському полі, а отже, визначають алгебри інваріантності такого рівняння Дірака. На цій основі Бозе симетрія спіна $s=(1,0)$ рівняння Дірака для вільного спінорного поля, доведена нещодавно в наших роботах, розширена на випадок рівняння Дірака, в якому врахована взаємодія із зовнішнім кулонівським полем. Релятивістський атом водню моделюється таким рівнянням Дірака. Отже, для релятивістського атома водню доведено як ферміонну, так і бозонну симетрію, що були відомі з наших робіт про інший випадок невзаємодіючого спінорного поля. Нові оператори симетрії знайдено на основі нових гамма-матричних зображень алгебр Кліффорда та $\mathrm{SO}(8)$, які також відомі з наших недавніх робіт. Приховані симетрії доведено як у канонічному представленні Фолді-Ваутхасена, так і у коваріантній моделі Дірака. Знайдені оператори симетрії, які є чисто матричними у представленні Фолді-Ваутхасена, стають нелокальними у моделі Дірака. 